\section{Revisão da Hiperglicemia Pós-Prandial e a Hipoglicemia no Controle do Diabetes Mellitus - O Papel da Insulina Lispro e Suas Pré-Misturas nos Picos e Vales}

\section{RESUMO}

A correção da hiperglicemia pós-prandial está ganhando importância cada vez maior em pacientes com diabetes tipo 1 (DM1) e tipo 2 (DM2) na redução do risco de morbidade e mortalidade. Estudos epidemiológicos têm indicado que há uma relação forte entre o grau de controle glicêmi$\mathrm{co}$, determinado pelos níveis de $\mathrm{HbAlc}$, e a freqüência de eventos cardiovasculares e mortalidade. Neste contexto, a hiperglicemia aguda tem sido implicada como um fator contribuinte para maior incidência de infarto do miocárdio, mortalidade cardíaca e na gênese de complicações microangiopáticas. Tratamento intensivo, geralmente envolvendo múltiplas injeções, e controle glicêmico estrito foram sugeridos pelos estudos DCCT, Kumamoto e UKPDS como o tratamento ideal em pacientes com DM para prevenir as complicações crônicas. Entretanto, o tratamento intensivo pode levar a uma maior incidência de episódios hipoglicêmicos, incluindo hipoglicemia severa, que pode ser um fator limitante para o atingimento de um bom controle metabólico. Agentes ideais para tratar o DM devem melhorar o controle glicêmico pós-prandial e global sem aumentar ou reduzindo o risco de hipoglicemia. Revisamos o perfil clínico de um análogo ultra-rápido da insulina humana, insulina lispro, e um novo análogo de longa duração insulina lispro NPL e as pré-misturas de insulina lispro e NPL (insulina lispro Mix25 e insulina lispro Mix50). As pré-misturas de lispro, assim com a insulina lispro, reduzem o incremento da glicemia pós-prandial comparado à insulina humana NPH ou pré-misturas de insulina humana em doses equivalentes. Adicionalmente, a insulina lispro, lispro Mix25 e lispro Mix50 reduzem o risco de hipoglicemia associado às preparações das insulinas humanas, principalmente os episódios noturnos. Insulina lispro Mix25 pode ser um bom tratamento para pacientes com DM1 ou DM2, que estejam atualmente em uso de uma pré-mistura de insulina humana de razão insulina ação curta/intermediária semelhante, de NPH isoladamente, ou com controle inadequado com agentes anti-diabéticos orais. (Arq Bras Endocrinol Metab 2001;45/5:423-432)

Unitermos: Diabetes tipo 1; Diabetes tipo 2; Hiperglicemia pós-prandial; Hipoglicemia; Insulina lispro; Mistura de insulina lispro; Insulina NPL.

\begin{abstract}
Correction of post-prandial hyperglycemia is increasingly becoming important to patients with diabetes type 1 (DM1) and type 2 (DM2) in order to reduce their risk of morbidity and mortality. Epidemiological studies have indicated that there is a strong relationship between the degree of glycemic control, determined by HbAlc levels, and the frequency of cardiovascular events and mortality. Acute hyperglycemia has been implicated as a contributed factor for increasing the incidence of myocardial infarction, cardiac mortality and in the genesis of the microangiopathic complications. Intensive treatment, usually involving multiple insulin injections, and tight glucose control have been suggested by trials as DCCT, Kumamoto and UKPDS as the ideal treatment in patients with DM in order to prevent chronic diabetic complications. However, intensive treatment may lead to an increased incidence of hypoglycemic episodes, including severe hypoglycemia, which can be a limiting factor for achievement of
\end{abstract}

revisão

\author{
Adolpho Milech \\ Antônio R. Chacra \\ Marcia J. Kayath
}

Disciplina de Diabetes e Nutrologia, Faculdade de Medicina da Universidade Federal do Rio de Janeiro, RJ (AM), Disciplina de Endocrinologia, Escola Paulista de Medicina, UNIFESP-EPM, SP (ARC) e Laboratório Eli Lilly do Brasil (MJK). 
optimal metabolic control. Ideal agents to treat DM should improve the postprandial and global glycemic control with no increased or less risk of hypoglycemia. We revise the clinical profile of a rapid-acting human insulin analogue, insulin lispro, and of a new long-acting analog insulin lispro NPL and fixed mixtures of insulin lispro and NPL (insulin lispro Mix25 and insulin lispro Mix50). Lispro mixtures, like insulin lispro itself, reduce the postprandial glucose rise compared to human insulin $\mathrm{NPH}$ or mixtures of similar ratios with equivalent doses of the insulin. Additionally insulin lispro, lispro Mix25 and Mix50 decrease the risk of hypoglycemia associated with human insulin preparations, particularly nocturnal episodes. Insulin lispro Mix25 may be a good therapy for DM1 or DM2 who are currently taking a similar ratio of short- to intermediate-acting insulin, using NPH alone, or experiencing inadequate control on oral antidiabetic agents. (Arq Bras Endocrinol Metab 2001;45/5:423-432)

Keywords: Diabetes type 1; Diabetes type 2; Postprandial hyperglycemia; Hypoglycemia; Insulin lispro; Insulin lispro mixture; Insulin NPL.

$\mathrm{O}$ PAPEL DA HIPERGLICEMA pós-prandial e da hipoglicemia como fatores limitantes importantes para se atingir o bom controle metabólico em pacientes com diabetes (DM) tem sido cada vez mais discutido. Nesta revisão discutimos as principais implicações clínicas destes dois fatores, principalmente no que se refere ao objetivo principal do tratamento do DM, qual seja, o controle rigoroso da glicemia. Os resultados do estudo Diabetes Control and Complications Trial (DCCT) demonstraram que uma melhora do controle glicêmico pode prevenir ou reduzir a severidade das complicações microvasculares em DMl (1). Também em DM2, o estudo Kumamoto e UKPDS demonstraram a mesma relação entre controle glicêmico e a prevenção de complicações microvasculares e macrovasculares (2-4). Na prática clínica, os objetivos principais do tratamento do DM têm sido a redução dos valores glicêmicos pré-prandiais, de jejum e da glico-hemoglobina $\left(\mathrm{HbA}_{\mathrm{lc}}\right)$.

Embora a $\mathrm{HbA}_{\mathrm{lc}}$ dê informações úteis a respeito do controle glicêmico global em um período relativamente longo ( 2 a 3 meses), esta medida não é muito sensível às variações diárias dos níveis de glicose sangüínea. Por isso, a $\mathrm{HbA}_{\mathrm{lc}}$ não reflete adequadamente mudanças agudas da glicemia pós-prandial. $\mathrm{O}$ estudo DCCT menciona que a $\mathrm{HbA}_{\mathrm{lc}}$ não é a mais completa expressão da hiperglicemia. O mesmo estudo menciona que outras características do controle glicêmico, que podem não estar refletidas na $\mathrm{HbA}_{\mathrm{lc}}$, podem adicionar ou modificar o risco de complicações. As excursões de glicemia pós-prandial são mencionadas como importantes no risco de desenvolvimento das complicações $(1,5)$. Em alguns estudos clínicos, o controle da hiperglicemia pós-prandial têm sido associado com a redução da $\operatorname{HbA}_{\mathrm{lc}}(6,7)$.

$\mathrm{Na}$ verdade, as concentrações de glicemia em jejum, pré-prandiais e pós-prandiais contribuem para o controle glicêmico global; portanto o controle dos três fatores devem melhorar tanto as complicações à curto prazo quanto à longo prazo do DM. Outros fatores implicados no desenvolvimento de complicações do DM não serão discutidos nesta revisão como a hipertensão, tabagismo e idade.

\section{HIPERGLICEMIA PÓS-PRANDIAL (“PICOS”)}

Após uma refeição, os níveis de glicose sangüínea se elevam e atingem um pico entre 30 e 60 minutos pós-prandiais. $\mathrm{O}$ aumento da glicose sangüínea é contrabalançado por uma liberação rápida de insulina (primeira fase), seguida de uma fase mais lenta de liberação de insulina (segunda fase), que proporciona atividade insulínica por uma duração mais prolongada. Os níveis de glicose sangüínea retornam aos níveis pré-prandiais em cerca de 2 a 4 horas (8). Em pacientes com DMl, os níveis de glicose sangüínea após uma refeição ou um teste de tolerância à glicose permanecem elevados por mais de 4 horas (8). Os esquemas terapêuticos com insulina regular nas refeições tentam assemelhar-se à resposta fisiológica da insulina às refeições. Porém, a insulina regular tem algumas limitações como aumento relativamente lento dos níveis de insulina, níveis do pico de insulina atingidos são inferiores ao pico de insulina atingidos fisiologicamente com a insulina endógena, com atividade máxima de redução da glicemia em 2 a 4 horas, enquanto que os níveis de glicemia máximos ocorrem cerca de 30 a 60 minutos após uma refeição. O tempo ótimo de administração pré-prandial é difícil de ser estabelecido, podendo haver hiperglicemia pós-prandial mesmo com atrasos pequenos de administração (9). Também do ponto de vista prático apresenta o inconveniente da necessidade de se programar com antecedência a administração da insulina (30 a 45 minutos pré-prandial).

Nos pacientes com DM2 inicial, estudos demonstraram que o problema do controle primário da glicose encontra-se nos níveis anormalmente elevados de glicose pós-prandial $(8,10,11)$. Portanto, os esforços são dirigidos para o controle dietético que constitui a base da terapia nesses pacientes. Entretanto, mesmo a rígida obediência à dieta alimentar não consegue impedir a hiperglicemia pós-prandial a longo prazo na maioria dos pacientes $(12,13)$. Nos pacientes com DM2, a resposta pós-prandial anormal é causada pela secreção alterada da 
insulina, por utilização periférica inadequada de glicose (resistência insulínica) e pela inabilidade de supressão adequada de produção de glicose hepática (7-10).

\section{HIPERGLICEMIA AGUDA E COMPLICAÇÕES CRÔNICAS DO DIABETES MELLITUS}

A hiperglicemia crônica tem sido correlacionada com o desenvolvimento de complicações crônicas do DM. Resultados de estudos recentes também indicam uma ligação entre a hiperglicemia aguda e o desenvolvimento de complicações do DM, como retinopatia e doença vascular periférica (14-43). O impacto potencial da hiperglicemia pós-prandial também tem sido discutido. Flutuações agudas da glicose sangüínea após as refeições contribuem significativamente para níveis glicêmicos cronicamente altos $(14,16)$. Além disso, a hiperglicemia pós-prandial pode desencadear vários mecanismos fisiológicos que resultam em homeostase alterada $(14,17,18,23,33,34)$. Vários estudos demonstraram uma ligação entre o controle da hiperglicemia pós-prandial com a redução das complicações do DM e/ou da mortalidade (14-16,20-28,36-39). Nos rins, a hiperglicemia aguda pode aumentar a taxa de filtração glomerular (TFG) em pacientes com DM (23). Este efeito é maior em pacientes com proteinúria do que naqueles sem proteinúria $(24,25)$. O aumento agudo da TFG ocorre rapidamente e continua enquanto a glicemia estiver elevada. Adicionalmente, a hiperglicemia pósprandial pode também estimular uma produção excessiva de colágeno pelas células renais, o que seria um importante contribuidor para o desenvolvimento da nefropatia diabética. Em um estudo retrospectivo em 52 pacientes com DMl, foi demonstrada uma relação entre os níveis de glicemia pós-prandial e o intervalo de tempo entre o início do DM ao desenvolvimento da nefropatia (24). No Estudo Kumamoto, estudo prospectivo envolvendo 110 pacientes com DM2, encontrou-se uma relação significante entre a hiperglicemia pós-prandial e a progressão da nefropatia $(2,19)$. Neste estudo, quando os níveis de glicemia pós-prandial aumentavam de $180 \mathrm{mg} / \mathrm{dl}$ para $260 \mathrm{mg} / \mathrm{dl}$, aumentava a taxa de piora da nefropatia em 6 vezes $(2,19)$.

Em relação à retinopatia, reconhece-se que a hiperglicemia aumenta o fluxo sangüíneo para a retina e que este evento tem um papel precoce no desenvolvimento e progressão da retinopatia diabética (2629). Vários estudos demonstraram que um bom controle glicêmico, determinado pela $\mathrm{HbA}_{1 \mathrm{c}}$, está associado à redução do risco de retinopatia $(1,2,4,19)$. No estudo Kumamoto uma relação específica entre a glicemia pós-prandial e retinopatia também foi demonstrada $(2,19)$. Não houve piora da retinopatia, quando a glicemia pós-prandial estava entre $140 \mathrm{mg} / \mathrm{dl}$ e $180 \mathrm{mg} / \mathrm{dl}$, entretanto, houve um aumento da taxa de piora da retinopatia em 10 vezes, quando os níveis médios de glicemia pós-prandial atingiam $260 \mathrm{mg} / \mathrm{dl}$. Uma relação semelhante foi identificada entre a progressão da retinopatia e nefropatia e níveis elevados de $\mathrm{HbA}_{\mathrm{lc}}$ e glicemia de jejum.

A hiperglicemia aguda também tem sido identificada como um fator de redução do limiar de dor, e poderia ter um papel no desenvolvimento da dor neuropática (30). A relação entre a hiperglicemia pósprandial e doença cardiovascular também tem sido discutida (31). Muitos pacientes com DM2 têm evidência de doença cardiovascular e ateroesclerose no momento do diagnóstico de sua doença (31-33). Isto ocorre devido a grande parte dos pacientes já terem tido algum grau de intolerância à glicose, manifestada por hiperglicemia pós-prandial por vários anos antes de progredir para DM franco (31-34). O Estudo de Intervenção de Diabetes (DIS) foi um estudo prospectivo em pacientes com DM2 recém diagnosticado (35). Neste estudo identificou-se que entre outros fatores, a glicemia pós-prandial era um fator de risco importante para o infarto do miocárdio e mortalidade nesta população. Neste mesmo estudo, níveis de glicemia de jejum elevados não foram fatores preditivos para aumento de risco de infarto do miocárdio ou mortalidade. Os níveis de hiperglicemia pós-prandias acima de $180 \mathrm{mg} / \mathrm{dl}$ foram particularmente importantes. Outros estudos sugerem uma relação entre hiperglicemia assintomática e risco de mortalidade em DM2: em uma totalidade de 17.285 homens com DM2, também demonstraram que os pacientes que tinham os níveis mais altos de glicemia pós-prandial de 2 horas e de glicemia de jejum tinham um risco significantemente maior de morte precoce (36-39). Níveis elevados de triglicérides e oxidação de LDL em DM2 têm sido relacionados à hiperglicemia pós-prandial e são considerados fatores de risco cardiovascular nestes pacientes (40-42). Embora a relação causa e efeito entre a glicemia pós-prandial e o desenvolvimento das complicações crônicas do DM permaneça em estudo, a importância deste parâmetro na fisiopatologia das complicações do DM e na contribuição nos níveis de $\mathrm{HbA}_{\mathrm{lc}}$ tem sido demonstrada em diversos estudos.

\section{HIPOGLICEMIA (“VALES”)}

O objetivo de redução de complicações crônicas do DM é geralmente atingido com esquema de tratamento intensivo com insulina de longa duração, associada 
a insulinas de curta duração (1-4). Porém, o estudo DCCT, Kumamoto e UKPDS demonstram que à medida que o controle glicêmico melhora, o risco relativo de hipoglicemia aumenta (1-3). O temor da hipoglicemia pode se tornar uma barreira na implementação de esquemas terapêuticos que tentam atingir um controle rigoroso da glicemia. Isto é válido tanto para os médicos como para os pacientes.

No estudo DCCT os eventos hipoglicêmicos graves ocorreram com uma taxa três vezes maior nos pacientes com DMl tratados intensivamente que o grupo em tratamento convencional (62 episódios por 100 pacientes/ano versus 19 episódios por 100 pacientes/ano, respectivamente), levando a mais hospitalizações por hipoglicemia nos pacientes tratados de forma intensiva (1). Nos estudos Kumamoto e UKPDS em pacientes com DM2, os eventos hipoglicêmicos também foram mais comuns no grupo em tratamento intensivo, seja em drogas orais ou insulina (2). É difícil calcular o risco estimado de hipoglicemia no DM2, entretanto, a morte por hipoglicemia é incomum no DM2, tendo havido uma morte por 1500 pacientes no estudo UKPDS (3). Mesmo assim nos estudos Kumamoto e UKPDS não se conseguiu a normalização da $\mathrm{HbA}_{1 c}$, em grande parte devido à hipoglicemia e por não terem buscado a otimização do controle glicêmico pós-prandial.

Os agentes ideais para o tratamento do DM devem melhorar o controle glicêmico global e pós-prandial sem aumentar o risco de hipoglicemia ou até ter um risco menor de hipoglicema em relação às outras terapêuticas. As metas terapêuticas desejadas estabelecidas pela Associação de Diabetes Americana são uma glicose plasmática de jejum ou pré-prandial menor que $120 \mathrm{mg} / \mathrm{dl}$, glicemia ao deitar menor que $140 \mathrm{mg} / \mathrm{dl} \mathrm{e}$ uma $\mathrm{HbA}_{\mathrm{lc}}$ inferior a 7\% (44). Recentemente, alguns agentes orais como as meglitinidas, e os análogos da insulina humana como a insulina lispro e aspart foram desenvolvidas com o objetivo de controlar a hiperglicemia pós-prandial. Nesta revisão estaremos abordando a insulina lispro, que é o análogo de insulina de ação ultra-rápida mais extensamente estudada até o momento. Adicionalmente, uma insulina NPL de ação intermediária foi desenvolvida a partir da insulina lispro com o objetivo principal de criar misturas de insulina lispro de ação rápida com uma insulina intermediária para uso diário a longo prazo e também será aqui descrita.

\section{INSULINA LISPRO}

Insulina lispro é um análogo da insulina humana desenvolvida por engenharia genética pela inversão dos aminoácidos prolina e lisina nas posições 28 e 29 da cadeia beta, resultando em uma insulina com seqüência $\operatorname{Lis}(\mathrm{B} 28) \operatorname{Pro}(\mathrm{B} 29)$ (45-48). Esta insulina tem menor tendência para auto-agregação no local de aplicação subcutânea, é absorvida mais rapidamente que a insulina humana regular e mimetiza o perfil fisiológico da insulina em resposta a uma refeição (47-48). Como resultado, ela tem um início de ação rápido (15 minutos), atinge um pico de ação mais precocemente (1 hora) e possui uma duração de ação mais curta (4 horas) (45-48). Estudos clínicos demonstraram que esta insulina monomérica tem efeitos benéficos, como a redução da incidência de episódios hipoglicêmicos (49-67) e um melhor controle glicêmico pós-prandial em pacientes com DM1 e DM2 (65-75). Além disso, a insulina lispro deve ser administrada imediatamente, ou, se necessário, após a refeição, mantendo ação eficaz (76-77).

Alguns estudos também mostraram uma redução significante da $\mathrm{HbA}_{\mathrm{lC}}$ em uso de insulina lispro (78-82). Estes estudos sugerem que, quando os pacientes são transferidos para insulina lispro, a estratégia de tratamento deve ser de ajustar tanto os esquemas de insulinização pré-prandiais quanto o esquema de doses e freqüência da insulina basal (78-84). Esta estratégia melhoraria o controle glicêmico global sem aumentar o risco de hipoglicemia.

A segurança do uso de insulina lispro, particularmente referente à taxa de hipoglicemia, também foi avaliada. Uma incidência menor de episódios hipoglicêmicos em pacientes em uso de insulina lispro, particularmente episódios noturnos em DMl e DM2 (49-67). Em uma meta-análise de hipoglicemia severa em diabéticos tipo 1 , a freqüência de hipoglicemia severa foi significativamente menor em pacientes em uso de insulina lispro, do que naqueles em uso de insulina regular (64). Quando se compara a freqüência de hipoglicemia leve entre insulina regular e lispro em um nível de $\mathrm{HbA}_{\mathrm{lc}}$ de $7,0 \%$, encontrou-se que o número de episódios hipoglicêmicos por paciente por mês foi mais baixo com a insulina lispro (85). Estudos prévios com insulina lispro também demonstraram preferência dos pacientes por insulina lispro, quando comparada à insulina humana regular e referem melhora da qualidade de vida dos pacientes (86-88). Em situações de alergia ou resistência à insulina humana, a insulina lispro também tem se mostrado como uma opção alternativa com boa resposta terapêutica (89-90). O uso de lispro na gestação em pacientes com DMl e DM2 e também em DM gestacional tem sido relatado, atingindo-se um bom controle glicêmico com menor incidência de hipoglicemia e sem diferença na incidência de anorma- 
lidades fetais quando comparada à insulina regular (9194). O uso da insulina lispro em bombas de infusão contínua subcutânea leva a um controle glicêmico melhor e mais estável e pode levar a uma menor necessidade de dose de insulina que a insulina regular (95-101).

\section{INSULINA LISPRO NPL}

A insulina lispro NPL é similar à NPH humana sendo composta por uma insulina de curta ação, que foi associada à protamina para prolongar sua duração de atividade (101-102). Entretanto, insulina NPL é diferente da NPH humana e de outras insulinas basais, porque forma misturas estáveis com insulina lispro, permitindo a associação de pré-misturas de insulina lispro (insulina lispro mix 25 ou mix50). Seu perfil de atividade é muito similar ao da NPH humana, sendo uma insulina de ação intermediária com uma duração de atividade de aproximadamente 10 a 14 horas (101103). Embora a insulina NPL tenha sido desenvolvida para permitir a formulação de misturas estáveis com insulina lispro, a insulina NPL pode ser utilizada isoladamente como uma insulina de ação intermediária. A atividade de insulina NPL é virtualmente idêntica à da NPH humana, mantendo o controle glicêmico noturno e glicemia de jejum de forma comparável à insulina NPH humana (103) (tabela 1). Os níveis de glicemia em jejum e à noite com misturas de insulina lispro e NPL são similares aos obtidos com uma automistura de NPH humana e insulina humana regular, entretanto, as misturas auto-preparadas de insulina lispro e NPL produziram melhor controle glicêmico global (medido por $\mathrm{HbA}_{\mathrm{lc}}$ ) e melhor controle glicêmico pós-prandial em pacientes com DMl e DM2 (104).

\section{INSULINA LISPRO MIX25 E MIX50}

Pré-misturas de insulina são freqüentemente utilizadas no tratamento do DM e consistem de combinações de

Tabela 1. Perfil de atividade farmacológica das insulinas humanas e análogos da insulina lispro $(54,109)$.

\begin{tabular}{|c|c|c|c|}
\hline & $\begin{array}{l}\text { Início de } \\
\text { ação }\end{array}$ & $\begin{array}{l}\text { Pico de } \\
\text { ação }\end{array}$ & $\begin{array}{c}\text { Duração de } \\
\text { ação }\end{array}$ \\
\hline Insulina NPH & $1-2$ horas & $6-12$ horas & 20-24 horas \\
\hline Insulina regular & 1 hora & $2-4$ horas & 6-8 horas \\
\hline Insulina $70 N / 30 R$ & 1 hora & $2-3$ horas & 10-14 horas \\
\hline Insulina lispro & 15 minutos & 1 hora & 4 horas \\
\hline Insulina NPL & $1-2$ horas & $6-12$ horas & 20-24 horas \\
\hline Insulina lispro Mix25* & 15 minutos & 1 hora & $10-14$ horas \\
\hline
\end{tabular}

* A insulina lispro Mix25 corresponde a 25\% de insulina lispro e $75 \%$ de insulina NPL. insulinas de ação curta e ação intermediária, geralmente administradas duas vezes ao dia. Estas formulações são aproximadamente $40 \%$ da insulina humana usada mundialmente, proporcionando maior conveniência ao paciente por melhorar a acurácia de dosagem e eliminar a necessidade de realizar a automistura (102). Segundo dados de 2001 do Instituto IMS Health, no Brasil o uso de pré-misturas é de aproximadamente $20 \%$ do total de insulinas usadas no mercado. O uso principal destas formulações é no DM2 e a pré-mistura mais utilizada é a $70 / 30$, seguida pela $80 / 20$ e $90 / 10$. Os especialistas preferem as auto-misturas nos pacientes com DMl e o uso de prémisturas nesta população é baixo.

As misturas com insulina regular apresentam algumas limitações, como o intervalo de tempo de injeção necessário antes de uma refeição, início de ação demorado e absorção lenta. As misturas de insulina lispro e de insulina NPH devem ser utilizadas imediatamente antes das refeições. Essas misturas não podem ser armazenadas durante longos períodos de tempo, pois a insulina NPH pode se misturar com a insulina lispro quando a solução é armazenada por tempo prolongado, diminuindo o perfil de ação rápida da insulina lispro. Por outro lado, as pré-misturas de insulina lispro e insulina NPL permitem formulação de misturas estáveis com insulina lispro (103).

A insulina lispro Mix25 e insulina lispro Mix50 são pré-misturas que contêm insulina lispro como insulina de ação rápida e insulina NPL como a parte de insulina de ação intermediária. A insulina lispro Mix25 corresponde a $25 \%$ de insulina lispro e $75 \%$ de insulina NPL, enquanto que a insulina lispro Mix50 corresponde a $50 \%$ de insulina lispro e $50 \%$ de insulina NPL. As pré-misturas de insulina humana mais utilizadas na prática clínica do mundo todo são aquelas com uma proporção de $70 / 30$ ou de $80 / 20$, respectivamente de insulina NPH e insulina regular e as diferenças clínicas entre estas duas pré-misturas de insulina não são consideradas significativas (101). Dessa forma, a proporção 75/25 de insulina lispro Mix25 foi a escolhida, por ser uma mistura dentro da variação das proporções mais utilizadas.

As pré-misturas de lispro melhoram o controle da glicemia pós-prandial com redução das excursões glicêmicas (104-110). Podem ser injetadas imediatamente antes de uma refeição ou, em circunstâncias especiais, logo após uma refeição (110). Estudos têm demonstrado que o tratamento com insulina lispro Mix25 pode reduzir o risco global de hipoglicemia, particularmente a hipoglicemia noturna. Nestes estudos, a dose ou do número de injeções, quando um 
paciente é transferido de misturas de insulina $\mathrm{NPH} /$ regular, ou das pré-misturas $70 / 30$ ou $80 / 20$ para lispro Mix25 é semelhante (106-110).

Os estudos clínicos de insulina lispro Mix25 foram planejados para comparar sua eficácia e segurança à insulina humana $\mathrm{NPH} e$ às misturas de insulina humana $80 / 20$ e 70/30. A maioria das misturas é usada atualmente em esquemas de aplicação de duas vezes ao dia, especialmente em pacientes com DM2. Por isso, nestes estudos a insulina foi utilizada duas vezes ao dia. Os pacientes foram instruídos a injetar lispro Mix25 imediatamente antes das refeições e a injetar insulina humana $80 / 20$ e $70 / 30,30$ a 45 minutos antes das refeições. Os resultados destes estudos em pacientes com DMl e DM2 mostraram que as excursões glicêmicas pós-prandiais são significantemente menores com lispro mix 25 comparadas às misturas de insulinas humanas (105-110).

A insulina lispro Mix25 e insulina humana $80 / 20$ foram administradas separadamente a 127 pacientes com DM1 ou DM2, por 3 meses, de forma randômica. Mix25 produziu um controle glicêmico geral adequado e um melhor controle glicêmico pósprandial comparado à insulina humana 80/20. A incidência e a taxa de hipoglicemia global foram similares entre os tratamentos. Contudo, a incidência de hipoglicemia noturna foi significativamente menor durante o tratamento com Mix25 (Mix25: 6\%, 20/80: $20 \%, \mathrm{p}=0,034)$ e o número de episódios hipoglicêmicos por paciente durante o dia foi significantemente menor durante tratamento com Mix25 (Mix25: 1,7 episódios, 80/20: 2,5 episódios, $\mathrm{p}=0,03)$. A insulina Lispro Mix25 e insulina humana 70/30 também foram administradas a 89 pacientes com DM2 duas vezes ao dia por 3 meses de forma randômica. As medidas de glicemia pós-prandial média de 2 horas após o café da manhã, e as medidas da glicemia pós-prandial média de 2 horas e excursões de glicose após o jantar, foram significantemente menores durante o tratamento com lispro mix 25 do que com 70/30 $(106,107)$.

A insulina lispro Mix50 foi administrada antes do café da manhã e Mix 25 antes do jantar por 3 meses, e insulina humana regular 50/50 antes do café da manhã e humana regular 70/30 antes do jantar por 3 meses em 100 pacientes com DMl ou DM2 de forma randômica. $\mathrm{O}$ controle glicêmico foi avaliado pela $\mathrm{HbAlc}$, perfis glicêmicos auto-monitorados, e pela freqüência e taxa de hipoglicemia em 30 dias. A lispro Mix50 reduziu significantemente a glicemia 2 horas após o café da manhã e a glicemia antes do almoço medida após o café da manhã. A insulina lispro Mix25 reduziu as excursões glicêmicas de 2 horas após o jantar comparada à 70/30 com um controle glicêmico noturno comparável baseado nas medidas da glicemia ao deitar, às 3:00 da madrugada, e em jejum. Ao final do estudo, o número de episódios hipoglicêmicos noturnos foi significantemente menor com lispro Mix25 (Mix25: 0,7 e com 70/30: $1,4 ; \mathrm{p}=0,037)(106,107)$.

O controle glicêmico global foi similar em ambos os tratamentos com níveis médios de $\mathrm{HbA}_{\mathrm{lc}}$ semelhantes ao final do tratamento, porém o risco global de hipoglicemia também se mostrou menor com lispro mix 25, particularmente a hipoglicemia noturna. Em comparação com a insulina humana 80/20 e 70/30, um número significantemente menor de pacientes experimentou el episódio de hipoglicemia noturna durante $\mathrm{o}$ tratamento com insulina lispro Mix25 $(106,107)$. O tratamento com lispro Mix25 também resultou em uma incidência global significantemente menor de episódios hipoglicêmicos $(106,107)$. As doses médias de insulina pela manhã e à noite foram similares para os dois tratamentos durante o estudo, o que demonstra que um paciente que estiver mudando de uma mistura de insulina $\mathrm{NPH} /$ regular para lispro Mix25 pode usar uma dose equivalente da nova terapia (105-111). Em outro estudo de refeição-teste lispro Mix25 também levou a uma menor excursão glicêmica pós-prandial de 2 horas quando comparada à mistura 70/30 e insulina NPH humana (105). Os investigadores do estudo concluíram que, nos pacientes com DM2, a elevação pós-prandial da glicose plasmática após a injeção de lispro Mix25 está mais próxima da fisiologia normal do que ocorreu com as outras duas insulinas testadas (105). O estudo de lispro Mix50 e lispro Mix25 também demonstrou uma incidência significantemente menor de hipoglicemia noturna com a terapia com as misturas de lispro em comparação com a terapia com misturas de insulina NPH (106). A insulina lispro mix 25 também resultou em menores excursões glicêmicas pós-prandiais com maior redução da hemoglobina glicosilada sem aumento nos episódios de hipoglicemia em comparação com a insulina NPH em 66 pacientes com DM2 (106). Ambas insulinas foram administradas duas vezes por dia antes do café e jantar e em doses equivalentes (109).

Pacientes que mais podem se beneficiar de insulina lispro Mix25 são aqueles pacientes que estejam usando uma mistura de insulina curta/intermediária ou apenas NPH, ou que estejam com controle inadequado com agentes antidiabéticos orais. A lispro Mix25 está também indicada em pacientes com DM2 que estejam iniciando o tratamento com insulina ou que não consigam preparar auto-misturas. Ao utilizar insulina lispro mix 25 poderão atingir um me- 
lhor controle glicêmico pós-prandial e global com risco semelhante ou menor de hipoglicemia.

\section{CONCLUSÕES}

Em comparação com as misturas baseadas em insulina NPH/regular, a terapia com insulina lispro Mix25 melhora o controle glicêmico pós-prandial, proporciona controle glicêmico global equivalente ou melhor em pacientes com DMl e DM2 e resulta em risco de hipoglicemia igual ou menor em comparação a pré-misturas de insulina humana ou a insulina NPH. A insulina lispro Mix25 é eficaz quando injetada imediatamente antes de uma refeição, proporcionando assim a praticidade de se administrar a insulina mais próximo às refeições.

\section{REFERÊNCIAS}

1. The Diabetes Control and Complications Trial Research Group. The effect of intensive treatment of diabetes on the development and progression of long-term complications in insulin-dependent diabetes mellitus. $\mathbf{N}$ Engl J Med 1993;329:977-86.

2. Onkubo Y, Kishikawa $H$, Araki $E$, et al. Intensive insulin therapy prevents the progression of diabetic microvascular complications in Japanese patients with non-insulin dependent diabetes mellitus: a randomized prospective 6-year study. Diab Res Clin Pract 1995;28:103-17.

3. Shichiri M, Kishikawa H, Ohkubo Y, Wake N. Long-term results of the Kumamoto study on optimal diabetes control in type 2 diabetic patients. Diabetes Care 2000;23:B21-9.

4. UK Prospective Diabetes Study (UKPDS) Group - Effect of intensive blood-glucose control with metformin on complications in overweight patients with type2 diabetes (UKPDS 34). Lancet 1998:352:854-65.

5. UK Prospective Diabetes Study Group. United Kingdom Prospective Diabetes Study 24: A 6-year, randomized, controlled trial comparing sulfonylurea, insulin, and metformin therapy in patients with newly diagnosed type 2 diabetes that could not be controlled with diet therapy. Ann Intern Med 1998; 128:165-75.

6. The Diabetes Control and Complications Trial Research Group. The relationship of glycemic exposure $\left(\mathrm{HbA}_{l \mathrm{c}}\right)$ to the risk of development and progression of retinopathy in the diabetes control and complications trial. Diabetes 1995; 44:968-83.

7. Kelley D, Mokan M, Veneman T. Impaired postprandial glucose utilization in non-insulin dependent diabetes mellitus. Metabolism 1994;43:1549-57.

8. Pampanelli S, Torlone E, Lalli $\mathrm{C}$, et al. Improved postprandial metabolic control after subcutaneous injection of a short-acting insulin analog in IDDM of short duration with residual pancreatic beta-cell function. Diabetes Care 1995; 18:1452-9.

9. Yki-Järvinen $\mathrm{H}$. Acute and chronic effects of hyperglycemia on glucose: implications for development of new therapies. Diabet Med 1997; 14:S32-S37.
10. Dineen SF. The postprandial state: mechanisms of glucose intolerance. Diabet Med 1997; 14:S19-S24.

11. Paolisso G, Giugliano D. Oxidative stress and insulin action: is there a relationship? Diabetologia 1996;39:357-63

12. Yki-Jarvinen $H$. Acute and chronic effects of hyperglycemia on glucose metabolism. Diabetologia 1990;33:579-85.

13. Ceriello A. Acute hyperglycemia and oxidative stress generation. Diabet Med 1997; 14:S45-S49.

14. Ceriello A. The emerging role of post-prandial hyperglycemic spikes in the pathogenesis of diabetic complications. Diabet Med 1998; 15:188-93.

15. De Veciana M, Major CA, Morgan MA, et al. Postprandial versus preprandial blood glucose monitoring in women with gestational diabetes mellitus requiring insulin therapy. N Engl J Med 1995;33:1237-41.

16. Avignon A, Radauceanu A, Monnier L. Nonfasting plasma glucose is a better marker of diabetic control than fasting plasma glucose in type 2 diabetes. Diabetes Care 1997:20:1822-6.

17. Diwadkar VA, Anderson JW, Bridges SR, Gowri MS, Oelgten PR. Postprandial low-density lipoproteins in type 2 diabetes are oxidized more extensively than fasting diabetes and control samples. Proc Soc Exp Biol Med 1999;222:178-84.

18. Verges B. The impact of regulation of postprandial glucose in practice. Diabetes Metab 1999:25:22-5.

19. Shichiri M, Kishikawa H, Ohkubo Y, Wake N. Long-term results of the Kumamoto Study on optimal diabetes control in type 2 diabetic patients. Diabetes Care 2000:23:B21-9.

20. Ceriello A. The post-prandial state and cardiovascular disease: relevance to diabetes mellitus. Diabetes Metab Res 2000; 16:125-32.

21. Lowe LP, Liu K, Greenland P, Metzger BE, Dyer AR, Stamler J. Diabetes, asymptomatic hyperglycemia, and 22year mortality in black and white men. Diabetes Care 1997;20(2): 163-9.

22. Donahue RP, Abbott RD, Reed DM, Yano K. Postchallenge glucose concentration and coronary heart disease in men of Japanese ancestry. Diabetes 1987;36:689-92.

23. Taylor RH. Post-prandial hyperglycaemia and diabetic complications. Practical Diabetes 1993; 10:S10-14.

24. Hasslacher C, Stech W, Wahl P, Ritz E. Blood pressure and metabolic control as risk factors for nephropathy in type 1 (insulin-dependent) diabetics. Diabetologia 1985;28:6-11.

25. Hasslacher C, Ritz E. Effect of control of diabetes mellitus on progression of renal failure. Kidney Int 1987;32:53-6.

26. Grunwald JE, Riva CE, Petrig GL, et al. Strict control of glycaemia: effects on blood flow in the large retinal vessels and in the macular microcirculation. Br J Ophthalmol 1995;79:735-41.

27. Grunwald JE, DuPont J, Riva CE. Retinal haemodynamics in patients with early diabetes mellitus. Br J Ophthalmol 1996;80:327-31.

28. Patel V, Rassam S, Newsom R, Wick J, Kohner E, et al. 
Retinal blood flow in diabetic retinopathy. Br Med J 1992;305:678-83.

29. Klein R, Klein BEK, Moss SE, Davis MD, DeMets DL. Glycosylated hemoglobin predicts the incidence and progression of diabetic retinopathy. JAMA 1988;260: 2864-71.

30. Perkins AT, Morgenlander JC. Endocrinologic causes of peripheral neuropathy. Postgrad Med 1997;102:81-106.

31. Haffner SM. Impaired glucose tolerance, insulin resistance, and cardiovascular disease. Diabet Med 1997; 14:S12-S18.

32. Gerstein HC. Glucose: a continuous risk factor for cardiovascular disease. Diabet Med 1997; 14:S25-S31.

33. Haller $\mathrm{H}$. Postprandial glucose and vascular disease. Diabet Med 1997; 14:S50-S56.

34. Temelkova-Kurktschiev TS, Koehler C, Henkel E, Leonhardt W, Fuecker K, Hanefeld M. Postchallenge plasma glucose and glycemic spikes are more strongly associated with atherosclerosis than fasting glucose or $\mathrm{HbA}_{1 \mathrm{c}}$ level. Diabetes Care 2000;23:1830-5.

35. Hanefeld M, Fischer S, Julius U, et al. Risk factors for miocardial infarction and death in newly detected NIDDM: the Diabetes Intervention Study 11 -year follow-up. Diabetologia 1996;39(12):1577-83.

36. Yano K, Kagan A, McGee D, Rhoads GG. Glucose intolerance and nine-year mortality in Japanese men in Hawaii. Am J Med 1982;72:71-80.

37. Donahue RP, Abbott RD, Reed DM, Yano K. Postchallenge glucose concentration and coronary heart disease in men of Japanese ancestry. Diabetes 1987;36:689-92.

38. Rodriguez BL, Curb JD, Burchfiel CM, Huang B, et al. Impaired glucose tolerance, diabetes, and cardiovascular disease risk factor profiles in the elderly. The Honolulu Heart Program. Diabetes Care 1996; 19:587-90.

39. Balkau B, Shipley M, Jarrett RJ, et al. High blood glucose concentration is a risk factor for mortality in middleaged nondiabetic men. Diabetes Care 1998;21:360-7.

40. Giugliano D, Ceriello A. Oxidative stress and diabetic vascular complications. Diabetes Care 1996; 19:257-67.

41. Van Dam PS, Van Asbeck BS, Erkelens DW, Marx JJM, Gispen WH, Bravenboer B. The role of oxidative stress in neuropathy and other diabetic complications. Diabet Metab Rev 1995; 11:181-92.

42. Rema M, Mohan V, Bhaskar A, Shanmugasundaram KR. Does oxidative stress play a role in diabetic retinopathy? Ind J Ophthalmol 1995;43:17-21

43. MacRury SM, Gordon D, Wilson R, et al. A comparison of different methods of assessing free radical activity in type 2 diabetes and peripheral vascular disease. Diabet Med 1993;10:331-5.

44. American Diabetes Association: Clinical Practice Recommendations 2001. Diabetes Care 2001:24:S33-S43.

45. Ciofetta M, Lalli C, Del Sindaco P, et al. Contribution of postprandial versus interprandical blood glucose to $\mathrm{HbAlc}$ in type 1 diabetes on physiologic intensive therapy with lispro insulin at mealtime. Diabetes Care $1999 ; 22: 795-800$.
46. Soonthornpun S, Rattarasarn C, Leelawattana R, Setasuban W. Postprandial plasma glucose: a good index of glycemic control in type 2 diabetic patients having near-normal fasting glucose levels. Diab Res Clin Pract 1999:46:23-7.

47. Howey DC, Bowsher RR, Brunelle RL, et al. (Lys(B28), Pro(B29))-human insulin: effect of injection time on postprandial glycemia. Clin Pharmacol Ther 1995;58:459-69.

48. Howey DC, Bowsher RR, Brunelle RL, Woodworth JR. (Lys(B28),Pro(B29))-human insulin: a rapidly-absorbed analogue of human insulin. Diabetes 1994;43:396-402.

49. Bohannon NJV. Benefits of lispro insulin. Postgrad Med 1997; 101:73-80.

50. Anderson JH, Brunelle RL, Keohane VA, et al. Mealtime treatment with insulin analog improves postprandial hyperglycemia and hypoglycemia in patients with noninsulin-dependent diabetes mellitus. Arch Int Med 1997; 157:1249-55

51. Vignati L, Anderson J, Iversen P. Efficacy of insulin lispro in combination with NPH human insulin, given twice per day in IDDM and NIDDM patients. Clin Ther 1997;19:1408-21.

52. Bastyr EJ $3^{\text {rd }}$, Johnson ME, Trautmann ME, Anderson JH $\mathrm{Jr}$, Vignati L. Insulin lispro in the treatment of patients with type 2 diabetes mellitus after oral agent failure. Clin Ther $1999 ; 21: 1703-14$.

53. Janssen MJ, Casteleijn S, Deville W, Popp-Snijders C, Roach $P$, Heine R. Nighttime insulin kinetics and glycemic control in type 1 diabetes patients following administration of an intermediate-acting lispro preparation. Diabetes Care 1997;20(12):1870-3.

54. Stiller R, Gudat U, Pfutzner A, Trautmann ME, Haslbeck M. Postprandial treatment with insulin lispro. Diabet Metab 1997:23:232-3.

55. Zinman B, Ross S, Campos RV, Strack T. Effectiveness of human ultralente versus NPH insulin in providing basal insulin replacement for an insulin lispro multiple daily injection regimen. A double-blind randomized prospective trial. The Canadian Lispro Study Group. Diabetes Care 1999;22(4):603-8.

56. Bolli GB. Rationale for using combinations of short-acting insulin analogue and NPH insulin at mealtime in the treatment of type 1 diabetes mellitus. J Pediatr Metab 1999; 12:737-44.

57. Torlone E, Fanelli C, Rambotti AM, Kassi G, Modarelli F, Di Vincenzo, et al. Pharmacokinetics, pharmacodynamics and glucose counterregulation following subcutaneous injection of the monomeric insulin analogue (Lys(B28), Pro(B29)) in IDDM. Diabetologia 1994;37:713-20.

58. ter Braak EW, Bianchi R, Erkelens DW. Faster, shorter and more profound action of (Lys(B28), Pro(B29)) human regular insulin analogue compared to regular insulin irrespective of the injection site (Abstract). Diabetes 1993;42:207A.

59. Heinemann L, Starke AAR, Heding L, Jensen I, Berger $M$. Action profiles of fast insulin analogues. Diabetologia 1990;33:384-6.

60. Anderson JH, Brunelle RL, Koivisto VA, Trautmann ME, Vignati L, DiMarchi $R$, et al. Improved mealtime treatment of diabetes mellitus using an insulin analogue. Clin Ther 1997; 19:62-72. 
61. Anderson JH, Brunelle RL, Kolvisto VA, Ptutzner A, Trautman ME, Vignati $L \mathrm{Jr}$, et al. Reduction of postprandial hyperglicemia and frequency of hypoglycemia in IDDM patients on insulin analogue treatment. Multicenter Insulin Lispro Study Group. Diabetes 1997;46:265-70.

62. Holleman F, Schmitt H, Rottiers R, Rees A, Symanowski S, Anderon JH for the Benelux-UK insulin lispro study group. Reduced frequency of severe hypoglycemia and coma in well-controlled IDDM patients treated with insulin lispro. Diabetes Care 1997;20:1827-32.

63. Pfutzner A, Kustner $E$, Forst $T$, Schulze-Schleppinghoff $B$, Trautmann M, Haslbeck M, et al. On behalf of the German Insulin lispro/IDDM study group: Intensive insulin therapy with insulin lispro in patients with type 1 diabetes reduces the frequency of hypoglycemic episodes. Exp Clin Endocrinol 1996; 104:25-30.

64. Brunelle RL, Llewelyn J, Anderson JH, Gale EAM, Koivisto VA. Meta-analysis of the effect of insulin lispro on severe hypoglycemia in patients with type 1 diabetes. Diabetes Care 1998;21:1726-31.

65. Jacobs MAJM, Keulen ETP, Kanc K, Casteleijn S, Scheffer $\mathrm{P}$, Devillé $\mathrm{W}$, et al. Metabolic efficacy of preprandial administration of Lys(B28), Pro(B29) human insulin analog in IDDM patients. A comparison with human regular insulin during a three-meal test period. Diabetes Care 1997;20:1279-86.

66. Campbell RK, Campbell LK, White JR. Insulin lispro: its role in the treatment of diabetes mellitus. Ann Pharm 1996;30:1263-71.

67. Wajchenberg BL, Chacra AR, Forti AC, Ferreira SRG, Oliveira O, Lopes CF, et al. Menor incidência de hipoglicemia noturna com o uso de insulina lispro comparada à insulina humana regular no tratamento de pacientes com diabetes tipo 1 Arq Bras Endocrinol Metab 2000;44:133-8.

68. Anderson JH, Brunelle RL, Keohane P, Koivisto VA, Trautmann ME, Vignati $L$, et al. Mealtime treatment with insulin analog improves postprandial hyperglycemia and hypoglycemia in patients with non-insulin-dependent diabetes mellitus. Arch Int Med 1997;157:1249-55.

69. Garg SK, Carmain JA, Braddy KC, Anderson JH, Vignati $\mathrm{L}$, Jennings $M K$, et al. Pre-meal insulin analogue insulin lispro vs. Humulin, insulin treatment in young subjects with type 1 diabetes. Diabet Med 1996;13:47-52.

70. Pampanelli S, Torlone E, Lalli C, Sindaco PD, Ciofetta M, Lepore M. Improved postprandial metabolic control after subcutaneous injection of a short-acting insulin analog in IDDM of short duration with residual pancreatic b-cell function. Diabetes Care 1995; 18:1452-9.

71. Feiglos MN, Thacker $\mathrm{CH}$, English J, Bethel MA, Lane JD. Modification of postprandial hyperglycemia with insulin lispro improves glucose control in patients with type 2 diabetes. Diabetes Care 1997;20:1539-42.

72. Torlone E, Pampanelli S, Lalli C, Del Sindaco P, Di Vicenzo A, Rambotti AM, et al. Effects of the short-acting insulin analog (Lys (B28), Pro(B29)) on postprandial blood glucose control in IDDM. Diabetes Care 1996; 19:945-52.

73. Jansson P-A, Ebeling P, Smith U, Conget I, Coves MJ, Gomis R. Improved glycemic control can be better maintained with insulin lispro than with regular insulin. Diab Nutr Metab 1998;1 1:194-9.
74. Bastyr EJB, Stuart CA, Brodows RG, Schwartz S, Graf CJ, Zagar A, et al. Therapy focused on lowering posprandial glucose, not fasting glucose, may be superior for lowering HbAlc. Diabetes Care 2000;23:1236-41.

75. Rutledge KS, Chase P, Klingensmith GJ, Walravens PA, Slover RH, Garg SK. Effectiveness of postprandial humalog in toddlers with diabetes. Pediatrics 1997;100:968-72.

76. Schernthaner G, Wein W, Sandholzer K, Equiluz-Bruck S, Bates $P$, Birkett MA. Postprandial insulin lispro. A new therapeutic option for type 1 diabetic patients. Diabetes Care 1998:21:570-3.

77. Rami B, Schober E. Postprandial glycaemia after regular and lispro insulin in children and adolescents with diabetes. Eur J Pediatr 1997; 156:838-40.

78. Melki V, Renard E, Lassmann-Vague V, Boivin S, Guerci B, Hanaire-Broutin $\mathrm{H}$. Improvement of $\mathrm{HbA}_{1 \mathrm{C}}$ and blood glucose stability in IDDM patients treated with lispro insulin analog in external pumps. Diabetes Care $1998 ; 21: 977-82$.

79. Ebeling P, Jansson PA, Smith U, Lalli C, Bolli GB, Koivisto $V A$. Strategies toward improved control during insulin lispro therapy in IDDM. Importance of basal insulin. Diabetes Care 1997:20:1287-9.

80. Ahmed AB, Mallias J, Home PD. Optimization of evening insulin dose in patients using the short-acting insulin analog lispro. Diabetes Care 1998;21:1162-6.

81. Ahmed AB, Home PD. Optimal provision of daytime NPH insulin in patients using the insulin analog lispro. Diabetes Care 1998:21:1707-13.

82. Sindako PD, Ciofetta M, Lalli C, Perriello, Pampanelli, Torlone $\mathrm{E}$, et al. Use of the short-acting insulin analogue lispro in intensive treatment of type 1 diabetes mellitus: importance of appropriate replacement of basal insulin and time-interval injection-meal. Diabet Med 1998;15: 592-600.

83. Ronnemaa T, Viikari J. Reducing snacks when switching from conventional soluble to lispro insulin treatment: effects on glycaemic control and hypoglycaemia. Diabet Med 1998; 15:601-7.

84. Strachan MWJ, Frier BM. Optimal time of administration of insulin lispro. Importance of meal composition. Diabetes Care 1998;21:26-31.

85. Lalli C, Ciofetta M, Del Sindaco P, et al. Long-term intensive treatment of type 1 diabetes with the short-acting insulin analog lispro in variable combination with NPH insulin at mealtime. Diabetes Care 1999:22:468-77.

86. Kotsanos JG, Vignati L, Huster W, Andreajasich C, Boggs $M B$, Jacobson AM, et al. Health-related quality-of-life results from multinational clinical trials of insulin lispro. Diabetes Care 1997:20:948-58.

87. Reviriego J, Millan M. Health-related quality of life and insulin lispro. A naturalistic approach. Diabetes Care $1998 ; 21: 1203-4$.

88. Grey M, Boland EA, Tamborlane WV. Use of lispro insulin and quality of life in adolescents on intensive therapy. Diabet Educ 1999;25:934-41.

89. Panczel P, Hosszufalusi N, Horvath MM, Horvath A. Advantage of insulin lispro in suspected insulin allergy. Allergy 2000;55(4):409-10. 
90. Raine $\mathrm{CH} 3^{\text {rd }}$, Krzyston MJ, Amr M, Hydrick L. Improvement in severe insulin resistance with frequent injections of lispro insulin. J Natl Med Assoc 1999:91(7):410-3.

91. RosenSG, Engel SS. Use of insulin lispro in pregnant women with diabetes mellitus. Diabetes 1998;47:A437.

92. Jovanovic L, llic S, Pettitt DJ, et al. Metabolic and immunologic effects of insulin lispro in gestational diabetes. Diabetes Care 1999;22(9):1422-7.

93. Bhattacharyya A, Brown S, Hughes SM, Vice PA. Lispro and regular insulin in pregnancy a comparison. $60^{\text {th }}$ American Diabetes Association Meeting 2000.

94. Calle-Pascual AL, Bagazgoitia J, Calle JR, Charro A, Maranes JP. Use of insulin lispro in pregnancy. Diab Nutr Metab 2000; 13:173-7.

95. Zinman B, Tildesley H, Chiasson JL, Tsui E, Strack T. Insulin lispro in CSII: results of a double-blind crossover study. Diabetes 1997;46(3):440-3.

96. Melki V, Renard E, Lassmann-Vague V, Boivin S, et al. Improvement of HbAlc and blood glucose stability in IDDM patients treated with lispro insulin analog in external pumps. Diabetes Care 1998;21(6):977-82.

97. Schmauss $S$, Konig $A$, Landgraf R. Human insulin analogue (LYS(B28), PRO(B29)\}: the ideal pump insulin? Diabet Med 1998; 15(3):247-9

98. Renner R, Pfutzner A, Trautmann M, Harzer O, Sauter K, Landgraf $R$. Use of insulin lispro in continuous subcutaneous insulin infusion treatment. Results of a multicenter trial. German insulin lispro-CSII Study Group. Diabetes Care 1999:22(5):784-8.

99. Johansson UB, Adamson UC, Lins PE, Wredling RA. Improved blood glucose variability, HbAlc insuman infusat (R) and less insulin requirement in IDDM patients using insulin lispro in CSII. The Swedish Multicenter Lispro Insulin Study. Diab Med 2000;26(3): 192-6.

100. Hanaire-Broutin $H$, Melki $V$, Bessières-Lacombe $S$, Tauber J-P. Comparison of continuous subcutaneous insulin infusion and multiple daily injection regimens using insulin lispro in type 1 diabetic patients on intensified treatment. A randomized study. The study group for the development of pump therapy in diabetes. Diabetes Care 2000;23(9): 1232-5.

101. DeFelippis MR, Bakaysa DL, Bell MA, et al. Preparation and characterization of a cocrystalline suspension of (LysB28,ProB29)-human insulin analogue. J Pharm Sci 1998;87(2): 170-6.

102. Turner HE, Matthews DR. The use of fixed-mixture insulins in clinical practice. Eur J Clin Pharmacol 2000;56:19-25.
103. Heise T, Weyer C, Serwas A, et al. Time-action profiles of novel premixed preparations of insulin lispro and NPL insulin. Diabetes Care 1998;21(5):800-3.

104. Strack T, Roach P, Anderson J - the NPL study group. Improved glycemic control with self-prepared mixtures of insulin lispro and a new sustained-release lispro formulation, NPL. Diabetes 1998;47:A62.

105. Koivisto VA, Tuominen JA, Ebeling P. Lispro Mix25 insulin as a premeal therapy in type 2 diabetic patients. Diabetes Care 1999:22:459-62.

106. Roach $\mathrm{P}$, Trautmann M, Arora V, Sun B, Anderson JH Jr. Improved postprandial blood glucose control and reduced nocturnal hypoglycemia during treatment with two novel insulin lispro-protamine formulations, insulin lispro mix25 and insulin lispro mix50. Mix50 study group. Clin Ther 1999;21(3):523-34.

107. Roach P, Yue L, Arora V. The Humalog Mix25 Study Group. Improved postprandial glycemic control during treatment with humalog Mix25, a novel protaminebased insulin lispro formulation. Diabetes Care 1999;22(8): 1258-61.

108. Malone JK, Woodworth JR, Arora V, Yang H, Campaigne $\mathrm{BN}$, Halle JP, et al. Improved postprandial glycemic control with Humalog Mix75/25 after a standard test meal in patients with type 2 diabetes mellitus. Clin Ther 2000;22(2):222-30.

109. Reviriego J, Herz M, Roach P. Humalog" Mix25' twice daily improves glycemic control compared to NPH twice daily in patients with type 2 diabetes. Diabetes 2000;49(1):A363-A364.

110. Hanusch U, Woodworth J, Roach P, Ristic S. Postprandial and preprandial administration of Humalog" Mix25' provide comparable glycemic control. Diabetologia 1999;42(1):A240, 1999 (Abstract 905).

111. Herz M, Arora V, Roach P for the Mix25 study group. Evaluation of dose conversion practices for Mix25, a manufactured fixed mixture of insulin lispro and NPL. Diabet Med 1999; 16:P60.

\section{Endereço para correspondência:}

Marcia Jeha Kayath

Eli Lilly do Brasil

Avenida Morumbi, 8264

04703-002 São Paulo, SP

e.mail: kayath@lilly.com 